\title{
The Associations of Epicardial Adipose Tissue With Coronary Artery Disease and Coronary Atherosclerosis
}

\author{
Se-Hong KIM, ${ }^{1} \mathrm{MD}$, Ju-Hye Chung, ${ }^{1} \mathrm{MD}$, Beom-June Kwon, ${ }^{2} \mathrm{MD}$, \\ Sang-Wook Song, ${ }^{1} \mathrm{MD}$, and Whan-Seok CHOI, ${ }^{1} \mathrm{MD}$
}

\begin{abstract}
SUMMARY
Epicardial adipose tissue (EAT) is presumed to play an important role in the development of coronary artery disease (CAD). The purpose of our study was to examine the influence of EAT volume measured by cardiac CT on the severity and presence of CAD. A total of 209 subjects (114 normal subjects and 95 patients with CAD) underwent cardiac and abdominal computed tomography (CT) scan before diagnostic coronary angiography. Pixels with a threshold range of -190 to -30 Hounsfield units were identified as EAT. CAGE $\geq 20$, CAGE $\geq 50$, and modified Gensini index were used to define the extent and severity of CAD. While there was no significant difference in BMI and WC between the two groups, the mean EAT volume was higher in the CAD group than in the normal subjects $\left(102.4 \pm 41.87 \mathrm{~cm}^{3}\right.$ versus $\left.125.36 \pm 47.64 \mathrm{~cm}^{3}, P<0.001\right)$. EAT was significantly associated with CAGE $\geq 20$, CAGE $\geq 50$, and Gensini score by linear regression analyses after adjusting for age, gender, smoking, and alcohol use. The severity of CAD increased linearly with each tertile increase in EAT volume $(P$ for trend $<0.05)$. Similarly, the coronary artery calcium $(C A C)$ score was also increased with each increase in EAT tertile $(P=0.002)$. In multivariate logistic regression models, EAT and VAT were significantly associated with the presence of CAD and CAC in age, gender, smoking, alcohol use, and BMI adjusted models. In conclusion, EAT volume measured by CT is associated with the presence and severity of CAD. EAT may give important information for risk evaluation in CAD. (Int Heart J 2014; 55: 197-203)
\end{abstract}

Key words: Computed tomography

$\mathrm{E}$ picardial adipose tissue (EAT) is the ectopic fat surrounding the heart, within the boundary of the pericardium. EAT shares the same embryological origin with abdominal visceral adipose tissue (VAT) from the splanchnopleuric mesoderm, ${ }^{1)}$ and the rate of lipolysis and lipogenesis is significantly higher in EAT than in other adipose tissues of the body. ${ }^{2)}$ Moreover, EAT is in direct contact with the major branches of the coronary vessels, and secretes numerous inflammatory cytokines. ${ }^{1,3,4)}$ Thus, EAT is presumed to play an important role in the development of coronary artery disease (CAD). ${ }^{1,5,6)}$

Although several investigators have reported that EAT may be related to the development of CAD, the definite role of EAT associated with CAD beyond VAT has not been determined. Furthermore, the correlation of EAT with the severity of CAD or coronary atherosclerosis is still being debated. ${ }^{7-10)}$ This controversy may result from the measurement method and study population. Earlier studies measured epicardial fat thickness using echocardiography or CT as an index of EAT mass. ${ }^{11-13)}$ However, given that the distribution of EAT is uneven around the heart, measurements of focal EAT thickness may not represent the epicardial fat burden. Indeed, it is still unclear which measurement location of EAT best reflects its $\mathrm{CAD}$ risk, and the two-dimensional measurements of EAT thickness showed low reproducibility compared with volumetric EAT measurement. ${ }^{14-17)}$ In contrast, volumetric measurement by computed tomography $(\mathrm{CT})$ has provided more accurate quantification of EAT. ${ }^{14,15)}$

The purposes of our study were: 1) to examine the influence of EAT volume measured by cardiac CT on the severity and presence of CAD; 2) to determine whether EAT is related to CAD compared with VAT; and 3) to evaluate the impact of EAT on coronary atherosclerosis.

\section{MeTHODS}

Subjects: A total of 209 consecutive subjects with suspected coronary artery disease were retrospectively evaluated from the outpatient clinic at St. Paul's Hospital, The Catholic University of Korea. All subjects underwent cardiac and abdominal computed tomography (CT) scans before diagnostic coronary angiography between September 2009 and November 2011. This study was approved by the Research Ethical Committee of the College of Medicine, the Catholic University of Korea.

Assessment of coronary angiographic data: All coronary angiography results were interpreted for the presence, extent and

From the Departments of ${ }^{1}$ Family Medicine and ${ }^{2}$ Internal Medicine, College of Medicine, The Catholic University of Korea, Seoul, Korea

Address for correspondence: Whan-Seok Choi, MD, The College of Medicine, The Catholic University of Korea 505 Banpo-Dong, Seocho-Gu Seoul 137-701, Korea. E-mail: iron1600@ catholic.ac.kr

Received for publication October 10, 2013. Revised and accepted October 17, 2013.

Released advance online J-STAGE May 7, 2014.

All rights are reserved to the International Heart Journal Association. 

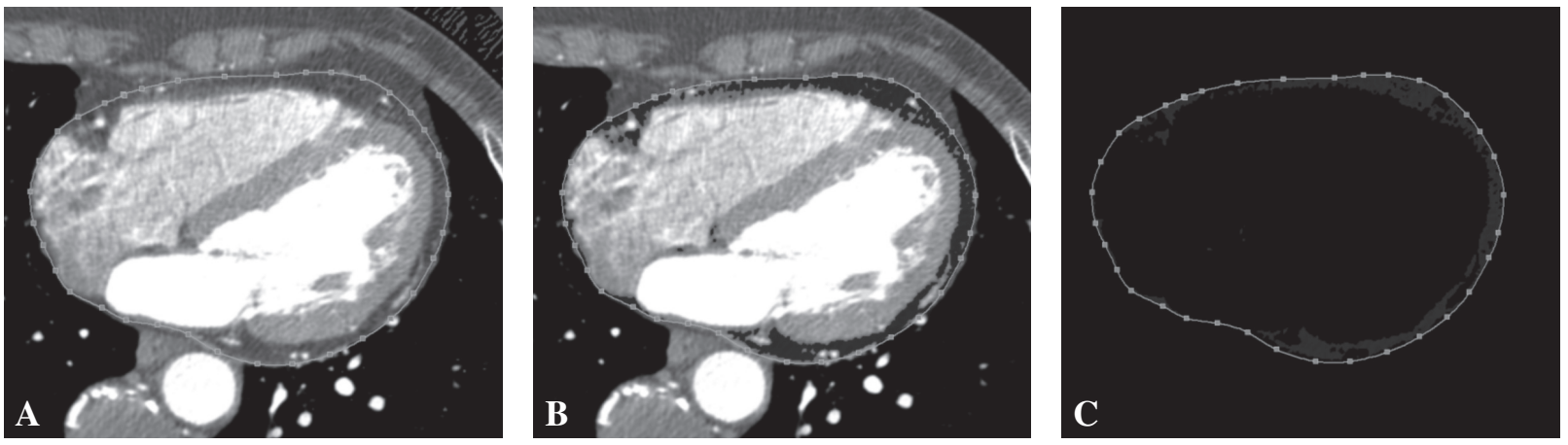

Figure 1. Epicardial adipose tissue (EAT) measurements. Segmentation of the axial images into EAT was performed by tracing the pericardium in axial slices (A). After region of interest (ROI) drawing, pixels with a threshold range of -190 to -30 Hounsfield units (HU) were identified as the EAT area (B, C).

severity of CAD by two experienced interventional cardiologists. "Any CAD" was defined as > 20\% luminal diameter stenosis in at least one major coronary artery or its branches, and "Significant CAD" as more than $50 \%$ narrowing in a coronary artery. Others were defined as normal coronary arteries. To define the extent of CAD, the total number of segments with a $50 \%$ or greater maximum stenosis was recorded as "coronary artery equal or greater than $50 \%$ (CAGE $\geq 50$ ) score". The total number of segments more than $20 \%$ was identified as CAGE $\geq 20$ score. $^{18)}$

The modified Gensini index was used to assess the severity of CAD. ${ }^{19,20)}$ The coronary vasculature was divided into 27 segments, and extent was determined by the number of affected segments. This scoring system grades luminal narrowing of the coronary arteries as 1 for $1-25 \%$ stenosis, 2 for $26-50 \%$ stenosis, 4 for $51-75 \%$ stenosis, 8 for $76-90 \%$ stenosis, 16 for $91-99 \%$, and 32 for total occlusion. For the location scores, 5 points were weighted for the left main coronary artery; 2.5 for the proximal left anterior descending (LAD) or left circumflex (LCX) artery; 1.5 for the mid segment LAD and LCX; 1 for the distal segment of LAD and LCX, first diagonal branch, first obtuse marginal branch, right coronary artery, posterior descending artery, and intermediate artery; and 0.5 for others. The points were then added and the total Gensini score of each patient was calculated.

CT Scan protocol: Contrast-enhanced coronary CT angiography was performed using a 16-slice MDCT scanner (LightSpeed 16, GE Healthcare, Milwaukee, WI, USA). An average of 250 contiguous slices were taken with an electrocardiography (ECG)-gated cardiac CT scanning protocol $(16 \times 0.63 \mathrm{~mm}$ slice collimation, $500 \mathrm{~ms}$ gantry rotation time, 0.275 pitch, 120 $\mathrm{kV}$ tube voltage, 300-470 mA tube current according to body size, image matrix of $512 \times 512$ pixels, temporal resolution 0.125 seconds, and slice thickness $0.625 \mathrm{~mm}$ ). Images were obtained at $70 \%$ of the cardiac cycle in the mid-diastolic phase, and intravenous $\beta$-blockers were administered for patients with heart rates $\geq 65$ beats $/ \mathrm{min}$. At the time of scanning, a bolus of $80 \mathrm{~mL}$ of contrast agent (Iopromide, Ultravist 370; BayerSchering, Berlin, Germany) was injected intravenously at a rate of $5 \mathrm{~mL}$ per second, followed by $50 \mathrm{~mL}$ of saline intravenously at a rate of $5 \mathrm{~mL}$ per second. To assess abdominal fat distribution, approximately 4-5 continuous transverse images $(120 \mathrm{kV}, 200 \mathrm{~mA}$, scanning time of 2 seconds, and slice thickness $5 \mathrm{~mm}$ ) were obtained at the L4-5 intervertebral space.

Adipose tissue measurement by CT: The EAT volume and
VAT area were measured by one experienced observer blinded to the clinical information and the coronary artery calcium score. All CT analyses were performed using a dedicated offline workstation (Rapidia, software ver. 2.8, Infinitt, Seoul, Korea).

EAT was defined as the adipose tissue between the outer surface of the myocardium and the visceral layer of the pericardium. We set the slice containing the center of the right pulmonary artery to be the upper slice limit and the last axial slice including any portion of the heart was chosen as the lower boundary. The segmentation of the axial images into EAT was performed on every fifth slice within this range using a semiautomated method. One expert reader set multiple seed points along the pericardium in each axial slice; these points were connected automatically to create a region of interest (ROI). After ROI drawing, pixels with a threshold range of -190 to -30 Hounsfield units (HU) was identified as the EAT area (Figure 1). The EAT volumes were calculated as the sum of the EAT volume of each slice from the superior to inferior boundary, in $\mathrm{cm}^{3}$. To quantify total EAT volume, we traced 30-40 slices per patient, depending on heart size. The reproducibility of EAT measurements was evaluated in a random sample of 20 participants by Pearson's correlation test. The intra- and interobserver reproducibilities for EAT volume were excellent (correlation coefficients 0.99 and 0.98 , respectively). The coronary artery calcium (CAC) was quantified in terms of the Agatston score, ${ }^{21)}$ and the presence of calcified coronary plaque was defined as an Agatston score $>0$.

Statistical analysis: Data were analyzed using SPSS software (ver. 13.0; SPSS Inc., Chicago, IL) and presented as means \pm standard deviation (SD). Values of fasting plasma glucose, HDL-cholesterol, systolic blood pressure, and triglycerides were highly skewed; thus, they were log-transformed for all analyses. The relationships of the extent and severity of CAD with multiple risk factors, including EAT, were presented as linear regression coefficients with $95 \%$ confidence intervals. One-way analysis of variance (ANOVA) was used to compare CAD severity according to EAT tertiles. Multiple logistic regression analysis was performed to assess the relationships of EAT with CAD and CAC. The odds ratio (OR) for the presence of CAD and CAC was based on a 1-SD increase in adipose tissue. A two-tailed $P<0.05$ was considered to indicate statistical significance. 
Table I. General Characteristics of Study Subjects $(n=209)$

\begin{tabular}{lccc}
\hline & Normal $(n=114)$ & CAD $(n=95)$ & $P^{*}$ \\
\hline Age (years) & $58.26 \pm 9.14$ & $60.77 \pm 9.16$ & 0.051 \\
Male & $50(43.86)$ & $45(47.37)$ & 0.889 \\
WC $(\mathrm{cm})$ & $87.18 \pm 9.60$ & $88.35 \pm 8.15$ & 0.366 \\
BMI $\left(\mathrm{kg} / \mathrm{m}^{2}\right)$ & $25.04 \pm 3.60$ & $24.27 \pm 4.79$ & 0.220 \\
Current smokers & $29(25.44)$ & $22(23.16)$ & 0.030 \\
Alcohol use & $49(42.98)$ & $32(33.68)$ & 0.144 \\
Diabetes mellitus & $24(21.05)$ & $36(37.89)$ & 0.012 \\
Hypertension & $66(57.89)$ & $72(75.79)$ & 0.013 \\
Metabolic Syndrome & $42(36.84)$ & $50(52.63)$ & 0.035 \\
Glucose (mg/dL) & $11.49 \pm 31.91$ & $131.00 \pm 62.76$ & 0.005 \\
HDL cholesterol (mg/dL) & $50.58 \pm 13.52$ & $44.89 \pm 12.16$ & 0.002 \\
Triglycerides $(\mathrm{mg} / \mathrm{dL})$ & $145.62 \pm 112.55$ & $137.52 \pm 90.23$ & 0.578 \\
LDL-cholesterol (mg/dL) & $109.91 \pm 37.21$ & $102.35 \pm 32.66$ & 0.130 \\
CRP $(\mathrm{mg} / \mathrm{dL})$ & $0.48 \pm 1.22$ & $1.01 \pm 2.29$ & 0.039 \\
EAT volume $\left(\mathrm{cm}^{3}\right)$ & $102.40 \pm 41.87$ & $125.36 \pm 47.64$ & 0.000 \\
VAT $\left(\mathrm{cm}^{2}\right)$ & $104.57 \pm 52.99$ & $125.50 \pm 56.75$ & 0.008 \\
SAT $\left(\mathrm{cm}^{2}\right)$ & $176.48 \pm 79.56$ & $147.56 \pm 56.63$ & 0.005 \\
Coronary calcium score & $26.75 \pm 64.98$ & $467.41 \pm 826.19$ & 0.000 \\
\hline Vile & &
\end{tabular}

Value are presented as the mean $\pm \mathrm{SD}$ (standard deviation) or $n(\%)$. * Statistical significances were tested by independent $t$-test or $\chi^{2}$ test. $n$ indicates number of persons; CAD, coronary artery disease; WC, waist circumference; BMI, body mass index; HDL, high-density lipoprotein; LDL, low-density lipoprotein; CRP, C-reactive protein, EAT, epicardial adipose tissue; VAT, visceral adipose tissue; and SAT, subcutaneous adipose tissue.

Table II. Associations of Multiple Risk Factors With the Extent of CAD

\begin{tabular}{|c|c|c|c|c|c|c|}
\hline & $\beta$ & SE & Standardized $\beta$ & $95 \% \mathrm{CI}$ & $P$ & $r^{2}$ \\
\hline \multicolumn{7}{|c|}{ Dependent variable: $C A G E \geq 20$ score } \\
\hline EAT volume $\left(\mathrm{cm}^{3}\right)$ & 0.007 & 0.003 & 0.190 & $0.224-2.219$ & 0.010 & 0.123 \\
\hline $\operatorname{VAT}\left(\mathrm{cm}^{2}\right)$ & 0.008 & 0.002 & 0.238 & $0.005-0.064$ & 0.001 & 0.1 \\
\hline $\operatorname{SAT}\left(\mathrm{cm}^{2}\right)$ & 0.000 & 0.002 & 0.005 & $-2.396-0.321$ & 0.953 & 0.067 \\
\hline $\mathrm{CRP}(\mathrm{mg} / \mathrm{dL})$ & 0.049 & 0.074 & 0.049 & $-0.291-0.752$ & 0.509 & 0.066 \\
\hline Glucose (mg/dL) & 1.222 & 0.505 & 0.177 & $0.357-5.001$ & 0.017 & 0.091 \\
\hline $\mathrm{WC}(\mathrm{cm})$ & 0.034 & 0.015 & 0.173 & $0.393-4.782$ & 0.021 & 0.095 \\
\hline HDL cholesterol (mg/dL) & -1.359 & 0.526 & -0.193 & $-2.396-0.321$ & 0.011 & 0.094 \\
\hline Triglycerides (mg/dL) & 0.231 & 0.264 & 0.066 & $-0.291-0.752$ & 0.384 & 0.063 \\
\hline $\mathrm{SBP}(\mathrm{mmHg})$ & 2.679 & 1.176 & 0.171 & $0.357-5.001$ & 0.024 & 0.095 \\
\hline $\mathrm{DBP}(\mathrm{mmHg})$ & 2.588 & 1.112 & 0.174 & $0.393-4.782$ & 0.021 & 0.096 \\
\hline \multicolumn{7}{|c|}{ Dependent variable: $\mathrm{CAGE} \geq 50$ score } \\
\hline EAT volume $\left(\mathrm{cm}^{3}\right)$ & 0.005 & 0.002 & 0.189 & $0.001-0.009$ & 0.010 & 0.088 \\
\hline $\operatorname{VAT}\left(\mathrm{cm}^{2}\right)$ & 0.005 & 0.002 & 0.193 & $0.001-0.008$ & 0.009 & 0.095 \\
\hline $\operatorname{SAT}\left(\mathrm{cm}^{2}\right)$ & 0.001 & 0.001 & 0.049 & $-0.002-0.004$ & 0.536 & 0.06 \\
\hline $\mathrm{CRP}(\mathrm{mg} / \mathrm{dL})$ & 0.069 & 0.053 & 0.096 & $-0.036-0.173$ & 0.197 & 0.06 \\
\hline Glucose (mg/dL) & 0.873 & 0.360 & 0.177 & $0.163-1.583$ & 0.016 & 0.083 \\
\hline $\mathrm{WC}(\mathrm{cm})$ & 0.029 & 0.010 & 0.206 & $0.008-0.049$ & 0.006 & 0.094 \\
\hline HDL cholesterol (mg/dL) & -0.786 & 0.382 & -0.155 & $-1.540-0.033$ & 0.041 & 0.07 \\
\hline Triglycerides (mg/dL) & 0.133 & 0.187 & 0.053 & $-0.237-0.503$ & 0.480 & 0.05 \\
\hline $\mathrm{SBP}(\mathrm{mmHg})$ & 1.824 & 0.742 & 0.171 & $0.362-3.287$ & 0.015 & 0.081 \\
\hline $\mathrm{DBP}(\mathrm{mmHg})$ & 0.026 & 0.010 & 0.198 & $0.007-0.046$ & 0.009 & 0.088 \\
\hline
\end{tabular}

Results are presented as linear regression coefficients with $95 \%$ confidence interval $(\mathrm{CI}), P$ and the coefficient of determination $\left(r^{2}\right)$ after adjusting for age, sex, smoking and alcohol use. CAGE $\geq 20$ score indicates coronary artery greater even than 20 score; and CAGE $\geq 50$ score, coronary artery greater even than 50 score.

\section{Results}

Baseline characteristics: The 209 subjects were divided into normal and CAD groups based on the presence of stenosis $>$ $20 \%$ in coronary arteries by diagnostic coronary angiography. Finally, 114 subjects were included in the normal group and 95 in the CAD group. Table I summarizes the baseline characteristics of the study participants with and without CAD. While there was no significant difference in BMI or WC between the groups, the mean EAT volume $(102.4 \pm 41.87$ versus $125.36 \pm$ $\left.47.64 \mathrm{~cm}^{3} ; P<0.001\right)$ and VAT area $(104.57 \pm 52.99$ versus $\left.125.5 \pm 56.75 \mathrm{~cm}^{2} ; P=0.008\right)$ were higher in the CAD group than in the normal subjects. SAT area was higher in the normal group $\left(176.48 \pm 79.56\right.$ versus $\left.147.56 \pm 56.63 \mathrm{~cm}^{2} ; P=0.005\right)$. Associations of EAT with the extent and severity of CAD: The associations between multiple risk factors and CAGE score are shown in Table II. EAT was significantly associated with CAGE $\geq 20$ and CAGE $\geq 50$ scores by linear regression analyses after adjusting for age, gender, smoking, and alcohol use $(P$ $<0.05)$. While significant positive associations were found for 
Table III. Multivariate Linear Regression Analysis for Epicardial Adipose Tissue With the Modified Gensini score

\begin{tabular}{|c|c|c|c|c|c|c|}
\hline & $\beta$ & SE & Standardized $\beta$ & $95 \% \mathrm{CI}$ & $P$ & $r^{2}$ \\
\hline EAT volume $\left(\mathrm{cm}^{3}\right)$ & 0.061 & 0.026 & 0.159 & $0.009-0.113$ & 0.022 & 0.202 \\
\hline $\operatorname{VAT}\left(\mathrm{cm}^{2}\right)$ & 0.036 & 0.023 & 0.112 & $-0.009-0.080$ & 0.118 & 0.032 \\
\hline $\operatorname{SAT}\left(\mathrm{cm}^{2}\right)$ & -0.018 & 0.019 & -0.075 & $-0.055-0.018$ & 0.323 & 0.025 \\
\hline $\mathrm{CRP}(\mathrm{mg} / \mathrm{dL})$ & 1.521 & 0.683 & 0.156 & $0.175-2.867$ & 0.027 & 0.039 \\
\hline Glucose (mg/dL) & 0.119 & 0.023 & 0.338 & $0.073-0.165$ & 0.000 & 0.128 \\
\hline $\mathrm{WC}(\mathrm{cm})$ & 0.152 & 0.134 & 0.080 & $-0.113-0.416$ & 0.260 & 0.029 \\
\hline HDL-cholesterol (mg/dL) & -0.187 & 0.092 & -0.144 & $-0.369-0.006$ & 0.043 & 0.029 \\
\hline Triglycerides (mg/dL) & -0.002 & 0.012 & -0.011 & $-0.025-0.021$ & 0.876 & 0.008 \\
\hline $\mathrm{SBP}(\mathrm{mmHg})$ & 0.058 & 0.088 & 0.047 & $-0.117-0.232$ & 0.515 & 0.019 \\
\hline $\mathrm{DBP}(\mathrm{mmHg})$ & 0.158 & 0.133 & 0.085 & $-0.104-0.420$ & 0.236 & 0.024 \\
\hline
\end{tabular}

Results are presented as linear regression coefficients with $95 \%$ confidence interval $(\mathrm{CI}), P$ and the coefficient of determination $\left(r^{2}\right)$ after adjusting for age, gender, smoking and alcohol use.

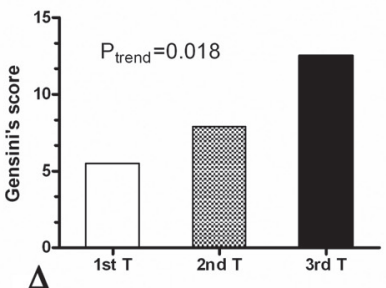

A

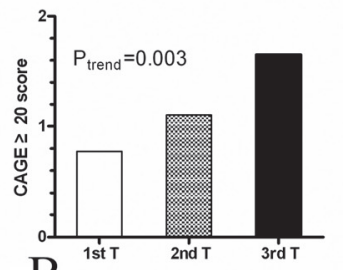

$\mathrm{B}$
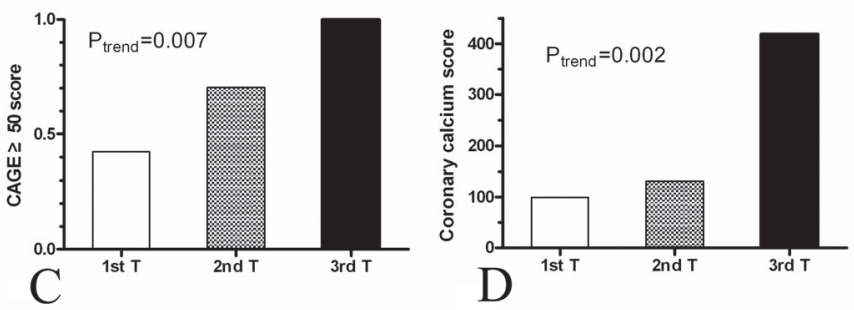

Tertiles of Epicardial adipose tissue

First tertile Second Tertile Third tertile

Figure 2. CAD severity according to tertiles of EAT volume. Gensini score (A), CAGE $\geq 20$ score (B), CAGE $\geq 50$ score (C), and coronary artery calcium score (D) increased linearly across the tertiles of EAT. CAGE $\geq 20$ indicates coronary artery greater even than 20 score; and CAGE $\geq 50$, coronary artery greater even than 50 score.

VAT, WC, SBP, DBP, and glucose, HDL-cholesterol was negatively related to the CAGE score. Linear regression analysis for EAT with the modified Gensini score is shown in Table III. Although the association between VAT and Gensini score was insignificant, EAT was associated with the Gensini score after adjusting for age, gender, smoking, and alcohol use $(P=$ $0.022)$. Significant associations with the Gensini score were also found for CRP, HDL-cholesterol, and glucose.

Comparison of the severity of CAD according to tertiles of EAT volume: We divided the subjects into tertiles according to EAT volume. A significant association between EAT tertiles with the severity of CAD was observed ( $P$ for trend $<0.05$, Figure 2 ). The severity of CAD increased linearly with each tertile increase in EAT volume: mean Gensini scores for each EAT tertile were $5.477 \pm 14.84,7.887 \pm 15.72$, and $12.507 \pm$ 20.59 , respectively $(P=0.018$, Figure $2 \mathrm{~A})$; mean CAGE $\geq 20$ scores for EAT tertiles were $0.773 \pm 1.46,1.101 \pm 1.67$, and $1.653 \pm 1.99$, respectively $(P=0.003$, Figure $2 \mathrm{~B})$; and mean CAGE $\geq 50$ scores for EAT tertiles were $0.424 \pm 0.82,0.704 \pm$ 1.35 , and $1.00 \pm 1.43$, respectively $(P=0.007$, Figure $2 \mathrm{C})$. Similarly, CAC score also increased with each increase in EAT tertile $(P=0.002$, Figure 2D).

Association of EAT with CAD and CAC: The association of EAT with the presence of CAD and CAC was assessed by multivariate logistic regression models (Table IV). EAT and VAT were significantly associated with $\mathrm{CAD}$, after adjusting for age and gender (Model 1). This association was unchanged and consistent after further adjustment for smoking, alcohol use, and BMI (Model 2 and Model 3). The OR between EAT and CAC was also significant in age, gender, smoking, alcohol use, and BMI adjusted models (OR 1.87; CI 1.15-3.04; $P=$ 0.011). However, the OR between VAT and CAC was insignificant after adjustment for BMI $(P=0.479)$.

\section{Discussion}

In this study, we examined the influence of EAT, measured by $\mathrm{CT}$, on CAD and CAC. EAT volume was higher in the CAD group than in normal subjects, and was significantly associated with CAD and CAC in multivariate logistic regression. Furthermore, EAT was associated with the extent and severity of CAD; CAGE $\geq 20, \mathrm{CAGE} \geq 50$, and Gensini scores increased linearly with the increase in EAT volume. Additionally, a significant association between EAT tertiles and the severity of CAC score was also observed. Taken together, these findings show that EAT is an independent risk factor for the presence and severity of CAD.

Earlier studies using echocardiography reported inconsistent results for the relationship between EAT and CAD according to study population. While a positive correlation was shown in nonobese subjects, ${ }^{12,22)}$ other studies of obese subjects $\left(\mathrm{BMI}>27 \mathrm{~kg} / \mathrm{m}^{2}\right)$ showed that EAT thickness was not associated with the severity of CAD or coronary calcium score. ${ }^{9,23)}$ These conflicting findings may be explained by the limitations of echocardiographic thickness measurements of EAT. 
Table IV. Multivariate Logistic Regression Analysis for Epicardial Adipose Tissue With CAD and Coronary Artery Calcium

\begin{tabular}{|c|c|c|c|c|c|c|}
\hline & \multicolumn{2}{|c|}{ Any CAD } & \multicolumn{2}{|c|}{ Significant CAD } & \multicolumn{2}{|c|}{$\mathrm{CAC}$} \\
\hline & OR $(95 \% \mathrm{CI})$ & $P$ & OR $(95 \%$ CI $)$ & $P$ & OR $(95 \%$ CI $)$ & $P$ \\
\hline \multicolumn{7}{|c|}{ Model 1: Age and gender adjusted } \\
\hline VAT & $1.43(1.06-1.92)$ & 0.019 & $1.43(1.05-1.94)$ & 0.021 & $1.59(1.09-2.30)$ & 0.015 \\
\hline EAT & $1.66(1.22-2.26)$ & 0.001 & $1.59(1.18-2.15)$ & 0.002 & $2.05(1.32-3.19)$ & 0.001 \\
\hline \multicolumn{7}{|c|}{ Model 2: Age, gender, smoking and alcohol adjusted } \\
\hline VAT & $1.50(1.08-2.08)$ & 0.015 & $1.51(1.08-2.11)$ & 0.015 & $1.59(1.07-2.37)$ & 0.021 \\
\hline EAT & $1.56(1.12-2.16)$ & 0.008 & $1.47(1.07-2.04)$ & 0.017 & $2.01(1.27-3.16)$ & 0.003 \\
\hline \multicolumn{7}{|c|}{ Model 3: Age, gender, smoking, alcohol and BMI adjusted } \\
\hline VAT & $1.85(1.26-2.72)$ & 0.002 & $1.81(1.24-2.65)$ & 0.002 & $1.03(0.94-1.13)$ & 0.479 \\
\hline EAT & $1.67(1.17-2.37)$ & 0.004 & $1.55(1.10-2.17)$ & 0.011 & $1.87(1.15-3.04)$ & 0.011 \\
\hline
\end{tabular}

Odds ratios (ORs) and 95\% confidence intervals (CIs) are presented as odds of CAD and CAC with a 1-SD increase in adipose tissue. CAD indicates coronary artery disease; CAC, coronary artery calcium; VAT, visceral adipose tissue; and EAT, epicardial adipose tissue.

Echocardiography does not provide a sufficient window around all cardiac segments, and has shown low reproducibility. ${ }^{14,16,17)}$ Furthermore, assessment of EAT by echocardiography is usually inadequate in obese patients due to poor acoustic windows. $^{24)}$

In contrast, computed tomography (CT) has provided accurate quantification of EAT amounts due to its high resolution compared with echocardiography. The volumetric measurement of EAT by cardiac CT is considered a validated method for the index of EAT mass, because many studies have demonstrated that EAT volume by CT is strongly associated with cardiometabolic risk factors ${ }^{5}{ }^{5}$ coronary calcium, ${ }^{25,26)}$ and CAD. ${ }^{27-30)}$ Thus, in this study, we measured EAT volume with $\mathrm{CT}$, and found that EAT volume was associated with the presence of CAD and CAC. In multivariate logistic regression, EAT was significantly associated with $\mathrm{CAD}$ after adjusting for age, gender, smoking, and alcohol use. The association between EAT and CAD was unchanged and consistent even after further adjustment for BMI. This suggests that EAT may be a contributing factor to $\mathrm{CAD}$, independent of overall adiposity. EAT was also associated with calcified coronary plaque, and the OR for CAC was higher in EAT than VAT, consistent with a previous report. ${ }^{5)}$ Interestingly, while there was no significant difference in BMI and WC between the groups, EAT volumes were higher in the CAD group than in normal subjects. This finding emphasizes that accurate quantification of EAT by CT is more important than simple anthropometric measurements such as weight, BMI, and WC. Consistent with our study, several studies have reported that EAT is related to the presence of $\mathrm{CAD}^{8,27,28)}$ and coronary atherosclerosis. ${ }^{10,26,29,30)}$

A few studies have reported on the association between EAT and the severity of CAD. Taguchi, et al. ${ }^{31)}$ reported that EAT measured by CT was correlated with the severity of CAD in nonobese male patients. Eroglu, et $a l^{32)}$ also reported that EAT thickness by echocardiography was associated with the severity of CAD. Consistent with these studies, our study further demonstrated that EAT volume measured by CT was associated with the severity of CAD. As with other known risk factors including VAT, WC, BP, FBS and HDL cholesterol, EAT was significantly associated with the extent and severity of CAD; CAGE $\geq 20, \mathrm{CAGE} \geq 50$, and Gensini scores were associated with EAT by linear regression analyses after adjusting for smoking, alcohol use, gender, and age. Additionally, a significant association between EAT tertiles and severity of $\mathrm{CAD}$ was found. The CAGE $\geq 20, \mathrm{CAGE} \geq 50$, and Gensini scores increased linearly with each tertile increase in EAT volume. Similarly, the CAC score also increased with each tertile increase in EAT volume. Taken together, these findings demonstrate that EAT is an independent risk factor for the presence and severity of CAD.

Although EAT was associated with the presence of CAD in most previous reports, the association with the severity of CAD has been controversial. ${ }^{7,9,23,33)}$ The lack of an association between EAT and CAD severity in these studies may be explained in the following aspects. First, no association between EAT and CAD severity was shown in two recent studies, ${ }^{7,9)}$ which did not include normal subjects. On the other hand, normal subjects and patients with minimal stenosis were included in our study. As a result, we found that the severity of CAD increased linearly with EAT volume, suggesting that EAT reflects a minimal change in early CAD. Second, most previous studies used a narrow definition of CAD, and thus did not account for the entire spectrum of CAD. ${ }^{7,8,10,34)}$ In these studies, CAD was defined only if stenosis was more than $50 \%$, otherwise as normal coronary arteries. In contrast, we defined the severity of CAD in more detail, using CAGE $\geq 20$, CAGE $\geq$ 50 , and the Gensini score, and found that severity of CAD increased simultaneously with EAT volume. Our findings provide more robust evidence for using EAT in assessing the severity of CAD.

The relationship between EAT and CAD in this study may be due to the paracrine effects of EAT. Perivascular EAT surrounding coronary vessels promotes the development of atherogenesis by direct diffusion of various cytokines and free fatty acid (FFA) into the vessel wall and the systemic circulation. ${ }^{1,2)}$ In contrast, intramyocardial coronary artery segments not in direct contact with EAT rarely contain atherosclerotic plaque. ${ }^{35)}$ Another possible explanation for the relationship between EAT and CAD is the enhanced inflammatory responses in EAT. Given that infiltration of inflammatory cells and expression of adipocytokines in EAT were strongly correlated with $\mathrm{CAD},{ }^{36-38)}$ chronic inflammation in EAT may influence the pathogenesis of coronary atherosclerosis. However, the definite pathogenesis between EAT and the coronary vessels regarding development of CAD needs further investigation.

Limitations: There are some limitations to this study. First, our findings may not be applicable to the general population, because the number of participants in this study was relatively small. Second, we performed a cross-sectional study, and we could not determine causality between EAT and CAD. How- 
ever, the study design has a strength in allowing a comprehensive assessment of angiographic CAD and volumetric EAT measurements. Further prospective studies are required to evaluate the relationship between changes in EAT and CAD over time.

Conclusion: We demonstrated that EAT volume measured by $\mathrm{CT}$ is associated with the presence and severity of CAD. EAT is a contributing factor to CAD independent of overall adiposity. Furthermore, EAT reflects the minimal change in early CAD. These findings suggest that EAT may provide important information for risk evaluation in $\mathrm{CAD}$, and may be useful as a predictor of CAD risk.

\section{ACKNOWLEDGMENT}

The authors wish to thank Moon-young Kim (INFINITT Healthcare Inc) and Dr. F. Saremi, MD (Department of Radiology, Keck School of Medicine, University of Southern California) for his review of our report.

\section{REFERENCES}

1. Sacks HS, Fain JN. Human epicardial adipose tissue: a review. Am Heart J 2007; 153: 907-17. (Review)

2. Şengul C, Özveren O. Epicardial adipose tissue: a review of physiology, pathophysiology, and clinical applications. Anadolu Kardiyol Derg 2013; 13: 261-5.

3. Mazurek T, Zhang L, Zalewski A, et al. Human epicardial adipose tissue is a source of inflammatory mediators. Circulation 2003; 108: 2460-6.

4. Baker AR, Silva NF, Quinn DW, et al. Human epicardial adipose tissue expresses a pathogenic profile of adipocytokines in patients with cardiovascular disease. Cardiovasc Diabetol 2006; 5: 1.

5. Rosito GA, Massaro JM, Hoffmann U, et al. Pericardial fat, visceral abdominal fat, cardiovascular disease risk factors, and vascular calcification in a community-based sample: the Framingham Heart Study. Circulation 2008; 117: 605-13.

6. Iacobellis G, Willens HJ. Echocardiographic epicardial fat: a review of research and clinical applications. J Am Soc Echocardiogr 2009; 22: 1311-9. (Review)

7. Gorter PM, de Vos AM, van der Graaf Y, et al. Relation of epicardial and pericoronary fat to coronary atherosclerosis and coronary artery calcium in patients undergoing coronary angiography. Am J Cardiol 2008; 102: 380-5.

8. Iwasaki K, Matsumoto T, Aono H, Furukawa H, Samukawa M. Relationship between epicardial fat measured by 64-multidetector computed tomography and coronary artery disease. Clin Cardiol 2011; 34: 166-71.

9. Chaowalit N, Lopez-Jimenez F. Epicardial adipose tissue: friendly companion or hazardous neighbour for adjacent coronary arteries? Eur Heart J 2008; 29: 695-7.

10. Djaberi R, Schuijf JD, van Werkhoven JM, Nucifora G, Jukema JW, Bax JJ. Relation of epicardial adipose tissue to coronary atherosclerosis. Am J Cardiol 2008; 102: 1602-7.

11. Iacobellis G, Assael F, Ribaudo MC, et al. Epicardial fat from echocardiography: a new method for visceral adipose tissue prediction. Obes Res 2003; 11: 304-10.

12. Jeong JW, Jeong MH, Yun KH, et al. Echocardiographic epicardial fat thickness and coronary artery disease. Circ J 2007; 71: 536-9.

13. Yorgun $\mathrm{H}$, Canpolat $\mathrm{U}$, Hazirolan $\mathrm{T}$, et al. Increased epicardial fat tissue is a marker of metabolic syndrome in adult patients. Int J Cardiol 2013; 165: 308-13.

14. Gorter PM, van Lindert AS, de Vos AM, et al. Quantification of epicardial and peri-coronary fat using cardiac computed tomogra- phy; reproducibility and relation with obesity and metabolic syndrome in patients suspected of coronary artery disease. Atherosclerosis 2008; 197: 896-903.

15. Bastarrika G, Broncano J, Schoepf UJ, et al. Relationship between coronary artery disease and epicardial adipose tissue quantification at cardiac CT: comparison between automatic volumetric measurement and manual bidimensional estimation. Acad Radiol 2010; 17: 727-34.

16. Fluchter S, Haghi D, Dinter D, et al. Volumetric assessment of epicardial adipose tissue with cardiovascular magnetic resonance imaging. Obesity (Silver Spring) 2007; 15: 870-8.

17. Saura D, Oliva MJ, Rodriguez D, et al. Reproducibility of echocardiographic measurements of epicardial fat thickness. Int J Cardiol 2010; 141: 311-3.

18. Schmermund A, Denktas AE, Rumberger JA, et al. Independent and incremental value of coronary artery calcium for predicting the extent of angiographic coronary artery disease: comparison with cardiac risk factors and radionuclide perfusion imaging. J Am Coll Cardiol 1999; 34: 777-86.

19. Gensini GG. A more meaningful scoring system for determining the severity of coronary heart disease. Am J Cardiol 1983; 51: 606.

20. Vlietstra RE, Kronmal RA, Frye RL, Seth AK, Tristani FE, Killip $\mathrm{T}, 3 \mathrm{rd}$. Factors affecting the extent and severity of coronary artery disease in patients enrolled in the coronary artery surgery study. Arteriosclerosis 1982; 2: 208-15.

21. Agatston AS, Janowitz WR, Hildner FJ, Zusmer NR, Viamonte M Jr, Detrano R. Quantification of coronary artery calcium using ultrafast computed tomography. J Am Coll Cardiol 1990; 15: 82732.

22. Ahn SG, Lim HS, Joe DY, et al. Relationship of epicardial adipose tissue by echocardiography to coronary artery disease. Heart 2008; 94: e7.

23. Nelson MR, Mookadam F, Thota V, et al. Epicardial fat: an additional measurement for subclinical atherosclerosis and cardiovascular risk stratification? J Am Soc Echocardiogr 2011; 24: 339-45.

24. Kessels K, Cramer MJ, Velthuis B. Epicardial adipose tissue imaged by magnetic resonance imaging: an important risk marker of cardiovascular disease. Heart 2006; 92: 962.

25. Ding J, Kritchevsky SB, Harris TB, et al. The association of pericardial fat with calcified coronary plaque. Obesity (Silver Spring) 2008; 16: 1914-9.

26. Greif M, Becker A, von Ziegler F, et al. Pericardial adipose tissue determined by dual source $\mathrm{CT}$ is a risk factor for coronary atherosclerosis. Arterioscler Thromb Vasc Biol 2009; 29: 781-6.

27. Oka T, Yamamoto H, Ohashi N, et al. Association between epicardial adipose tissue volume and characteristics of non-calcified plaques assessed by coronary computed tomographic angiography. Int J Cardiol 2012; 161: 45-9.

28. Sarin S, Wenger C, Marwaha A, et al. Clinical significance of epicardial fat measured using cardiac multislice computed tomography. Am J Cardiol 2008; 102: 767-71.

29. Alexopoulos N, McLean DS, Janik M, Arepalli CD, Stillman AE, Raggi P. Epicardial adipose tissue and coronary artery plaque characteristics. Atherosclerosis 2010; 210: 150-4.

30. Bettencourt N, Toschke AM, Leite D, et al. Epicardial adipose tissue is an independent predictor of coronary atherosclerotic burden. Int J Cardiol 2012; 158: 26-32.

31. Taguchi R, Takasu J, Itani Y, et al. Pericardial fat accumulation in men as a risk factor for coronary artery disease. Atherosclerosis 2001; 157: 203-9.

32. Eroglu S, Sade LE, Yildirir A, et al. Epicardial adipose tissue thickness by echocardiography is a marker for the presence and severity of coronary artery disease. Nutr Metab Cardiovasc Dis 2009; 19: 211-7.

33. Sironi AM, Petz R, De Marchi D, et al. Impact of increased visceral and cardiac fat on cardiometabolic risk and disease. Diabet Med 2012; 29: 622-7.

34. Wang TD, Lee WJ, Shih FY, et al. Association of epicardial adipose tissue with coronary atherosclerosis is region-specific and independent of conventional risk factors and intra-abdominal adi- 
posity. Atherosclerosis 2010; 213: 279-87.

35. Robicsek F, Thubrikar MJ. The freedom from atherosclerosis of intramyocardial coronary arteries: reduction of mural stress -- a key factor. Eur J Cardiothorac Surg 1994; 8: 228-35.

36. Shimabukuro M, Hirata Y, Tabata M, et al. Epicardial adipose tissue volume and adipocytokine imbalance are strongly linked to human coronary atherosclerosis. Arterioscler Thromb Vasc Biol
2013; 33: 1077-84

37. Hirata Y, Kurobe H, Akaike M, et al. Enhanced inflammation in epicardial fat in patients with coronary artery disease. Int Heart $\mathrm{J}$ 2011; 52: 139-42.

38. Hirata Y, Tabata M, Kurobe H, et al. Coronary atherosclerosis is associated with macrophage polarization in epicardial adipose tissue. J Am Coll Cardiol 2011; 58: 248-55. 